\title{
Energy Dispersive X-ray technique applied to fatigue fracture surfaces oxidation to provide information on growth rate
}

\author{
Iñigo Hernandez ${ }^{1, *}$, Edward A. Saunders ${ }^{2}$, and Iñaki Madariaga ${ }^{1}$ \\ ${ }^{1}$, Industria de Turbopropulsores, SAU, Materials \& Processes Department, Parque tecnológico nº300, 48170 Zamudio, Spain \\ ${ }^{2}$, Rolls-Royce plc, Materials - Failure Investigation, Bristol BS34 7QE, United Kingdom
}

\begin{abstract}
Gas turbine components are strongly affected by cyclic loading during operation, which makes fatigue development the most significant cause of failure or cracking within them. The nature and growth rate of that fatigue is dependent on the stress experienced at a particular area of a part, the temperature, the material properties and the applied loading frequency. Various techniques, such as striation and/or band counting can, in some cases, provide information on fatigue life. However, often such techniques are rendered useless by lack of formation or obliteration by damage and/or oxidation of such features. Once a crack has initiated and is exposed to the engine atmosphere, the level of oxidation and other contaminant elements observed on the crack surface will evolve with time, and, correspondingly, crack depth. Element distribution analysis by an Energy Dispersive x-ray Spectroscopy (EDS) technique applied along a fatigue fracture surface could help to understand the evolution of the crack with time.
\end{abstract}

Using EDS, an experimental procedure was carried out to ascertain a perceived measured percentage of oxygen plus other additional chemical species at a large number of stations along the length of a fatigue crack. The analysis was performed from the initiation site to the end of the propagation area, and also within the final rupture zone which had less time exposed to air and gasses compared to the fatigue system. An exercise of comparison between that progression in oxidation across cracked in-service components versus perceived measured oxidation level analyses on trial fracture surfaces exposed in atmospheric oven conditions at high temperatures for a number of different durations was performed. The obtained results provided information on specific questions concerning the fatigue propagation life of the in-service components; thereby amplifying the use of the EDS technique in this aspect of the materials forensic field.

\section{Introduction}

An explanation of a gas turbine engine broken blade through fatigue propagation is described herein. Some examples of blade release investigations may be found in [1-6]. Evaluation of the root cause is assisted through usage of the EDS technique, helping understanding of the evolution of the crack with time.The EDS technique and fundamentals is described in [7-10].

Strip inspection of an engine that suffered an event during running, revealed damage to different components, including a broken blade as one of the most significant finding. Therefore, the understanding of the blade fracture was considered essential in terms of knowing what the primary failure of the turbine was.

Fracture surface evaluation of the blade partially assembled in the disc showed evidence of a fatigue mechanism, but in spite of that, certain doubts about when the crack was produced arose. In particular, there was uncertainty about whether that blade was the primary root cause and produced the rest of the damage in the turbine, or it was a consequence of the failure of another component.

\section{Fracture surface assessment}

\subsection{Visual inspection}

The fracture surface of the blade was produced near the inner shroud of the component, about $10-15 \%$ of the aerofoil span (see figure 1).

The fracture of the blade started in the suction side of the aerofoil profile close to the trailing edge (see Fig.2). An area approximately $11 \mathrm{~mm}$ long with thin thickness (about $0,45 \mathrm{~mm}$ ) in that particular region of the surface was detected. That related to a thickness reduction in that area from nominal of around $65-70 \%$, whilst the remainder of the section in the blade was close to nominal (see figure 2).

Corresponding author: inigo.hernandez@itpaero.com; 


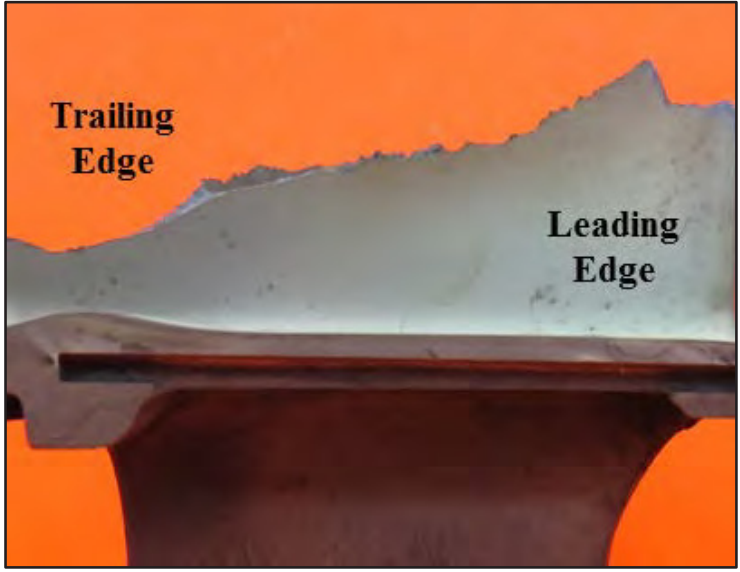

Fig. 1.Pressure side view of the broken blade.

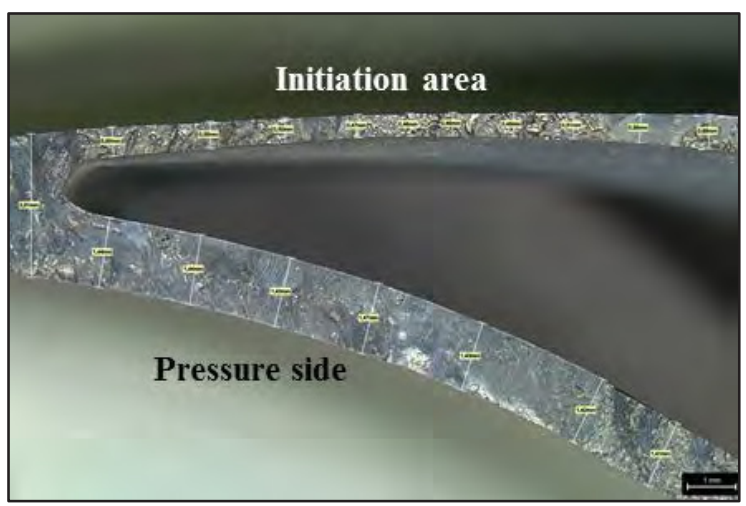

Fig. 2.Initiation in a thickness reduction area at suction side.

\subsection{Fractographic assessment}

The appearance of the blade fracture surface was mainly flat, except in the leading edge area and the pressure side adjacent to the LE that was more irregular and rough.

Beach and river marks typical of fatigue mechanisms were observed on the fracture surface in both directions from the initiation area of the crack (see Fig.3). Additionally, fatigue striations were observed only at the end of the fracture surface (see Fig.4). The features observed were commensurate with fatigue propagation in predominantly high cycle modes.

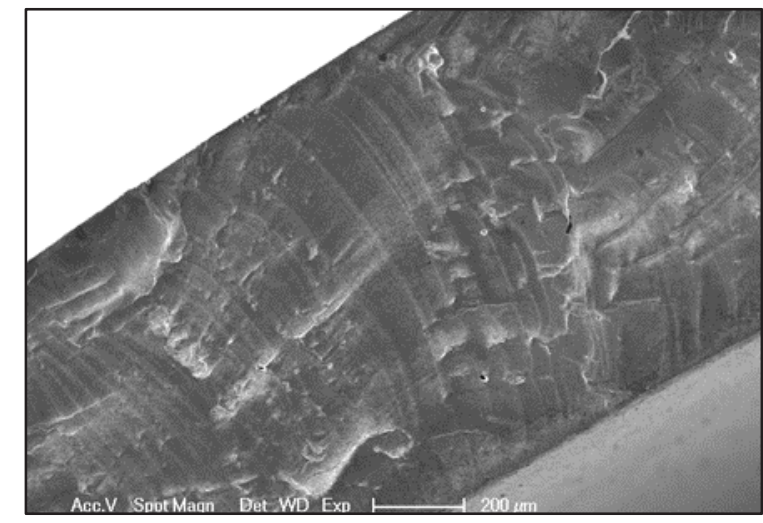

Fig. 3. Beach and river marks observed along the fatigue propagation area.

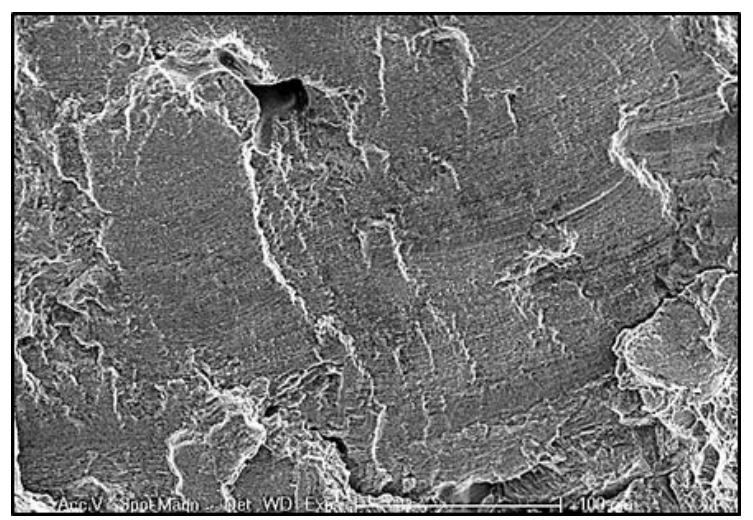

Fig. 4. Fatigue striations at the end of the propagation area, close to leading edge static fracture surface.

Summarizing, the assessment of the broken blade fracture surface showed an initiation area at the suction side with a thin thickness comparing to the rest of the aerofoil walls. Fatigue propagated from that position in both directions, toward leading and trailing edges, and going forward through a partial area of the pressure side. Accordingly, the fracture surface of the leading edge area and part of the pressure face showed ductile fracture features typical from static and sudden breakage (see Fig. 5).

\section{Energy Dispersive Spectroscopy on fracture surface}

\subsection{EDS on blade fracture surface}

The crack surface is exposed to the engine gas environment and subjected to oxidation and other contaminant elements (amongst other elements, deposits of silicon and phosphorous are often detected on the surface of turbine components). The level of that contamination evolves with the exposure time and with crack depth. Therefore, an Energy Dispersive x-ray Spectroscopy (EDS) technique applied along a fatigue fracture surface could help to understand the evolution of the crack with time.

The purpose of the analysis was to obtain the percentage of oxygen and silicon at plenty of stations along the length of the fatigue fracture surface. The analysis was carried out from the end of the fracture surface to the initiation area, including chemical analysis at the final rupture (the static one) which would have been exposed to the engine-running environment for less time than the fatigue propagation area. 


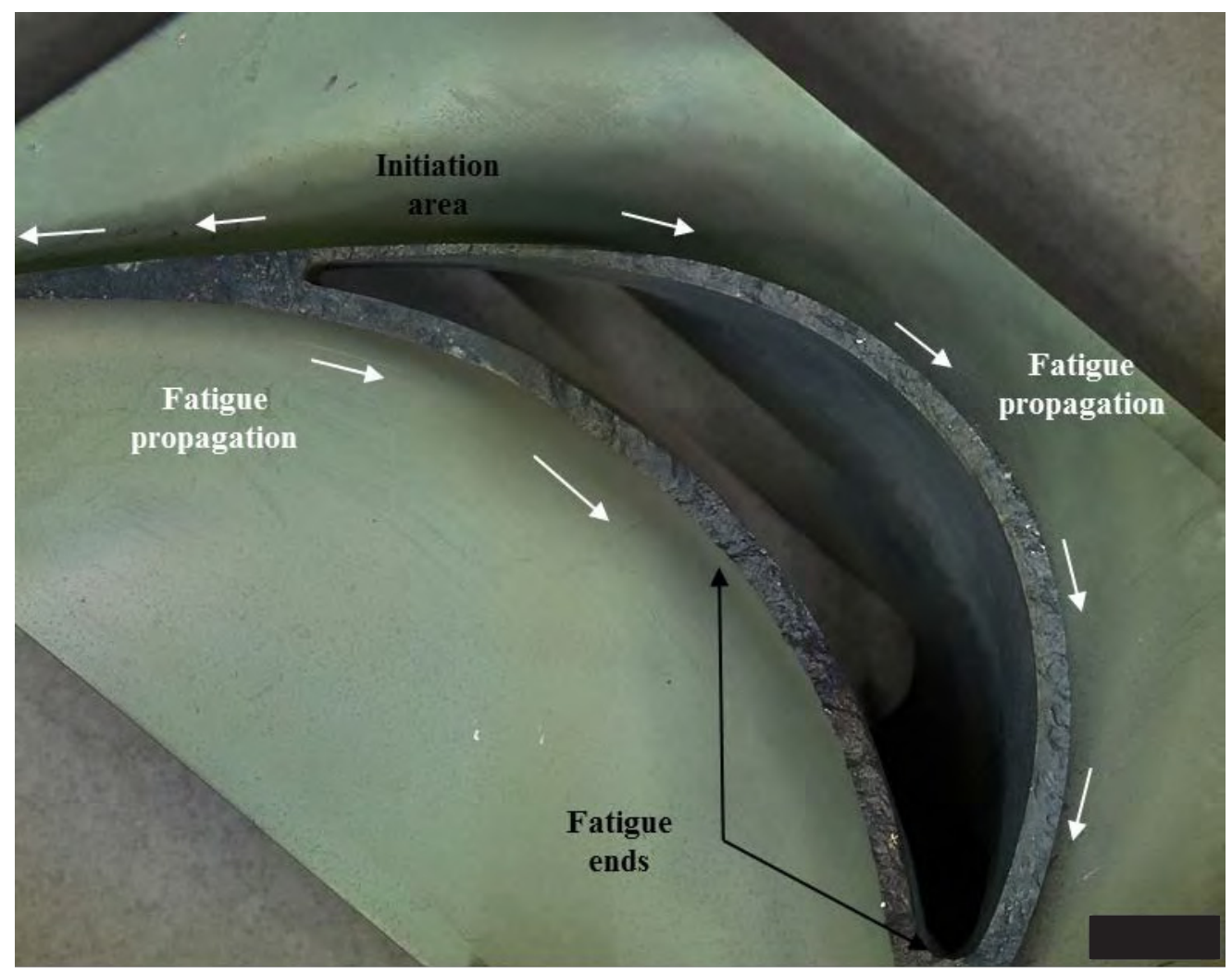

Fig. 5. General view of blade fracture surface.

Measurements started in a static fracture region in the area close to the leading edge, with "point zero" being the transition point from the end of the fatigue system to static fracture at the suction side of the aerofoil.

Those analyses were carried out under low pressure/ high vacuum conditions, using a low accelerating potential (voltage). Spot size and working distance were slightly modified to achieve the same test conditions along the fracture surface (keeping Counts and Dead Time parameters within the same ranges along the extension of the analysed area).

A clear increasing oxygen percentage was observed starting from the end of the fatigue propagation limit to the origin of the crack (see Fig. 6). Silicon, from surface contamination, showed a similar behaviour, although the quantity of this element was lower. Oxidation, therefore, had been evolving with time and in this particular way was "measurable".

It is recognised that the oxygen percentage measured is not absolute, as it depends on the $\mathrm{x}$-ray escape depth/volume ('bowl') with respect to the thickness of the oxide layer and how much underlying parent material that encompasses [7-10]. That escape depth/volume is governed in part of the applied accelerating voltage. By use of a relatively low voltage (eg. $10 \mathrm{kV}$ ) this sensitises the surface (an oxide layer) to a greater extent as the escape volume is smaller, so encompasses a greater proportion of the oxide layer.

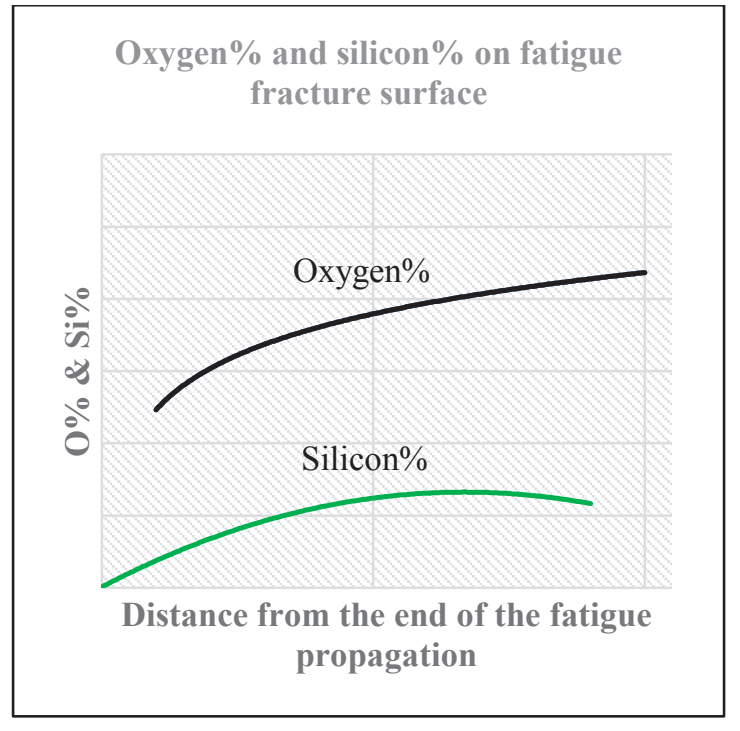

Fig. 6.Oxygen and silicon percentage along fatigue fracture surface.

Accordingly, in the current paper, the absolute oxygen percentage has not been sought, nor has it been the intent to measure an oxide thickness by this methodology. However, the work is intended to demonstrate a changing/increasing oxygen percentage that relates to a thickness as a comparative exercise. 


\subsection{EDS on treated test samples}

Fresh fracture faces (in tensile overload) were generated by breaking open blade material of the same alloy as the blade (IN713LC), as initially unaffected by temperature. These fracture faces were then over-exposed to the typical temperature as seen in service in the engine over nearly 20 different exposure times from 1 hour to 800 hours (at 1 atmosphere).

EDS analysis of those samples after each heat treatment was performed to generate an oxygen percentage value under the same analysis conditions as used on the blade fatigue fracture surface. It is appreciated that there will be variance in oxidation on directly-exposed tensile overload samples compared to that seen on a growing fatigue crack, but it was found that the oxygen percentage measured was similar enough to provide an estimate of the growth time of the fatigue crack - as a comparative measure (as described below).

The results of the tests again showed a clear increase in $\mathrm{O} \%$ content as the sample is exposure for longer times, although the level of $\mathrm{O} \%$ content after long exposed times was found to be lower than the $\mathrm{O} \%$ content calculated in areas near the fatigue initiation in the broken blade (see Fig. 7).

It was observed that, the tendency of increasing $\mathrm{O} \%$ content as the fracture is exposed for longer times at relatively high temperature is similar to the change in $\mathrm{O} \%$ content observed on the fracture surface of the broken blade. The difference of the $\mathrm{O} \%$ content could be explained by several reasons:

- The difference of the surface being exposed under a clean atmosphere in a furnace during the tests compared to under gas path conditions in the production engine.

- There been other contaminants in the analysis of the broken blade surface $(\% \mathrm{Si}$ and $\% \mathrm{P}$ content) not observed in the tests carried out in the laboratory.

- The differences between the flatness of the surface of the broken blade associated with fatigue and the fracture caused in the lab (static fracture) that was rougher.

- The dispersion of the results observed during the different analyses conducted in samples treated under the same condition.

- It was found in general for both studies that the EDS O\% results exhibited a logarithmic trend, as it is observed in general oxidation theory - in that oxide thickness development under temperature exposure shows a logarithmic trend with time $[11,12]$.

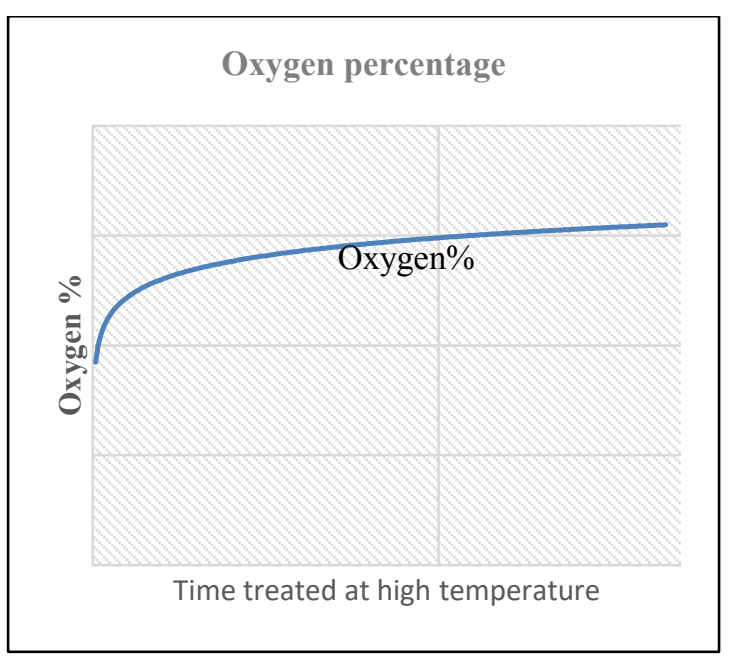

Fig.7.Oxygen percentage on test samples treated long exposure time.

\section{Summary}

As soon as a crack initiates it was exposed to the turbine atmosphere, and as a consequence of that the conditions of the surface change with air exposure with time, and, correspondingly, crack depth.

Energy Dispersive x-ray Spectroscopy (EDS) technique applied along a fatigue fracture surface has helped understand the evolution of the oxide layer on the crack surface with time for the oxygen percentage increases as the analysed surface is more exposed to the air providing the propagation took a significantly large number of hours. The level of contaminant silicon is another indicator of the time that a surface is exposed to the air.

A similar (logarithmic) oxygen percentage increase was also observed doing the same EDS exercise using laboratory-broken fracture surface samples treated from 1 hour to more than 800 hours at high temperature.

The sequence of component part failure in this engine/turbine was thereby established - in that the blade fracture was primary. This is because the blade fatigue surface had taken hours to develop (as recorded by the oxidation curve), and no rapidly if it had been instigated through failure of another part in the turbine.

The EDS results provided information concerning the fatigue propagation life of the in-service components, amplifying the use of this technique in the failure analysis field.

\section{References}

1. Y.-S. Choi and K.-H.Lee., Investigation of blade failure in gas turbine, 1969-1974, Journal of mechanical Science and Technology 24 (10) (2010)

2. X. Xu and Z. Yu, An investigation on the failed blades in a locomotive turbine, Engineering Failure Analysis, 14 (7) (2007) 322-1328.

3. W. Wang, F. Xuan, K. Zhu and S. Tu, Failure analysis of the final stage blade in steam turbine, Engineering Failure Analysis, 14 (4) (2007) 632-641. 
4. Z. Mazur, A. Hernandez-Rossette and R. GarciaIllescas, Investigation of the failure of the L-O blades, Engineering Failure Analysis, 13 (8) (2006) 13381350.

5. Z. Mazur, A. Luna-Ramırez, J. A. Juarez-Islasb and A.Campos-Amezcua, Failure analysis of a gas turbine blade made of Inconel 738LC alloy, Engineering Failure Analysis, 12 (13) (2005) 474486.

6. T. Carter, Common failures in gas turbine blades, Engineering Failure Analysis, 12 (2) (2005) 237-247.

7. JI Goldstein, DE Newbury, JR Michael, NWM Ritchie, JHJ Scott, DC Joy. Scanning Electron Microscopy and X-ray Microanalysis. Springer, US, 2003

8. JC Russ, M Ashby Frs, R Kiessling, J Charles. Fundamentals of Energy Dispersive X-ray Analysis. Elsevier, 1984. https://doi.org/10.1016/B978-0-40811031-0.50002-X

9. Oxford Instruments Energy Dispersive Spectroscopy (EDS) Operating Manual.

$\mathrm{http}: / /$ research.engineering.ucdavis.edu/cnm2/wpcontent/uploads/sites/11/2017/04/EDXSSOP_033017.pdf

10. B. Hafner. Energy Dispersive Spectroscopy on the SEM: A Primer.

http://www.charfac.umn.edu/instruments/eds_on_se $\mathrm{m} \_$primer.pdf

11. IM Ritchie. A logarithmic law of metal oxidation which is controlled by a surface reaction. The Philosophical Magazine: A Journal of Theoretical Experimental and Applied Physics, Volume 19, (1969) - Issue 158

12. HH Uhlig, Initial oxidation rate of metals and the logarithmic equation, Acta Metallurgica, Volume 4, Issue 5, (1956), Pages 541-554

Acknowledgement to Richard Hanlon from the Rolls-Royce Derby Failure Investigation team as he came up with the idea originally. 